\title{
Research on evaluation of funding utilization rate of colleges
}

\author{
Zhirui Liang \\ North China Electric Power University, Huadian Road 689,071000 Baoding, China \\ Zhirui_Liang@126.com
}

Keywords: Funding Utilization Rate, Grey Correlation Analysis, Factor Analysis

\begin{abstract}
Investment from grant organizations is a main constituent part of the income of colleges, and the metric always used to select candidate schools is the funding utilization rate. We refer total income of school as cost index and indicators (the number of degrees and credit hours) as efficiency index which reflects the effect of funding. Entering these indices into Grey Correlation Analysis Model, we can get the funding utilization rate of school.
\end{abstract}

\section{Introduction}

We are discussing the problem basing on the problem C in MCM 2016.

The Goodgrant Foundation is a charitable organization that wants to help improve educational performance of undergraduates attending colleges and universities in the United States. To do this, the foundation intends to donate a total of $\$ 100,000,000$ to an appropriate group of schools per year, and they want to invest to these schools which have the highest return on investment.

We define the return on investment as promotion on comprehensive evaluation of invested schools. The comprehensive evaluation index implies two aspects as tuition payment and teaching achievement. So colleges with higher return on investment are the colleges with higher funding utilization rate.

National Center on Education Statistics, which maintains an extensive database of survey information on nearly all post-secondary colleges and universities in the United States, and the College Scorecard data set which contains various institutional performance data. They release a list of potential candidate schools and provide us lots of related data. To simplify our work, we assume that prospective institutions to invest are entirely derived from the Table "IPEDS UID for Potential Candidate Schools" provided by the two organizations.

\section{Modeling and Solving}

\section{Step 1: Seek the modeling method.}

We hope to obtain the funding utilization $\eta$ of each school by the equation $\eta=\frac{\Delta S}{\Delta F}$, where $\Delta F$ is a unit funding aid to a school, and $\Delta S$ is the effect of fund. Nonetheless, difficulty in precisely measuring $\Delta S$ negates the direct equation solution, forcing us to seek other solutions. With regard to each school, performance has an ambiguous relationship to financial incomes, causally enlightening us on the idea of fuzzy mathematics to set up the Grey Correlation model to evaluate a system. We set investment funding and effect received as indices with lower investment funding and better effect received representing higher funding utilization.

\section{Step 2: Determine evaluation index by factor analysis}

Using factor analysis, we change six index which are used to evaluate colleges into two principal factors. Both two principal factors make sense in reality. The first principal factor, defined as Payment Factor (P), mainly scores through Net Price, Percentage of Pell Students, 3-year repayment rate and Completion rate, which reflects student payment for tuition and loan; and the second principal factor, defined as Achievement Factor (A), mainly scores through Median earnings and Proportion of non-low-income, which reflects the competence after graduation. 


\section{Step 3: Determine measurements.}

Given that total fund and its effect are two types of metrics for assessment, we try best effort to find all-sided indices reflecting the operation of a school considering that total fund of each school can be directly gained from the dataset.

The expenditure of total fund a school receives per year mainly includes basic operating expense and development expense. While a portion of the development expense primarily promotes whole performance of school(embracing the level of A and P defined in step 2), the rest of development expense is proposed to expand teaching scale by adding the number of degrees awarded (replace this index with graduation rate for non-degree awarded colleges) and the number of credit hours (Considering that federal government allocates its fund mainly by degrees awarded, credit hours and the mean faculty salary, it is the number of degrees awarded and the number of credit hours that ameliorate obviously with grant[1])

To sum up, we determine all indices in this model which consist of total incomes of school, the number of degrees awarded (or the graduation rate), the number of credit hours, achievement factor A and payment factor P. Data about these indices can be attained directly or indirectly from the dataset provided[2].

\section{Step 4: Calculate the indices.}

(1) Total incomes of school

While we can get funding resources and total incomes of colleges directly, modification is necessary to assess the utilization rate as we take into consideration the impact Consumer Price Index (CPI) has on funding. The higher CPI relates to larger demand for funding to get the same performance.

Assuming that original income of a school is $F^{\prime}$, then the modified one is

$$
F=\frac{F^{\prime}}{C P I} \text {. }
$$

So, same $F$ can generate same effect even in different years after modification.

(2) The number of degrees awarded (or the graduation rate)

The expense of a degree awarded varies in accordance with the degree types, so does the social contribution. Therefore, we have to put appropriate weight on the number of different degrees. Providing that a school award $B$ bachelor's degrees (or associate's degrees), $M$ master's degrees and $D$ doctor's degrees per year, meanwhile the proportion of person with above three degrees respectively is $a, b$ and $c$, then weight on each degree ought to move inversely to the corresponding proportion of person. The equation can be expressed as

$$
\text { Sumdegree }=\frac{1}{a} B+\frac{1}{b} M+\frac{1}{c} D
$$

(3) The number of credit hours

We can get the number of credit hours for each school per year directly by dataset.

(4) Achievement factor A and payment factor $P$

The two factor can be separately calculated in reference to the expressions in Step 2.

\section{Step 5: Set up the Grey Correlation method[3].}

(1) Make data normalization.

Supposing $m$ schools and $n$ metrics, we separate metrics into benefit-oriented and cost-oriented, and normalize all data corresponding to metrics type.

For benefit-oriented metric, $b_{i j}=\frac{a_{j}^{\max }-a_{i j}}{a_{j}^{\max }-a_{j}^{\min }}$.

For cost-oriented metric, $b_{i j}=\frac{a_{i j}-a_{j}^{\min }}{a_{j}^{\max }-a_{j}^{\min }}$.

Where $b_{i j}$ is normalized variable $\mathrm{i}, a_{i j}$ is pre-normalized variable $\mathrm{i}, a_{j}^{\min }$ and $a_{j}^{\max }$ are respectively minimum and maximum variables. 
(2) Ensure the objective sequence and the reference sequence.

The objective sequence can be expressed as $X_{i}=\left\{X_{i}(k) \mid k=1,2, \ldots, n\right\}$ while the reference sequence as $X_{0}=\left\{X_{0}(k) \mid k=1,2, \ldots, n\right\}$.

(3) Determine weight on each metric.

We identify weight vector as $w=\left[w_{1}, \ldots, w_{n}\right]$ where $w_{k}(k=1,2, \ldots, n)$ is weight on $k^{\text {th }}$ metric. Here we assume weight on each metric is equal, that is, $w_{1}=w_{2}=\ldots=w_{n}$.

(4) Calculate the grey relational coefficient.

$$
\xi_{i}(k)=\frac{\Delta_{\min }+\rho \Delta_{\max }}{\Delta_{i k}^{(0)}+\rho \Delta_{\max }}
$$

Where $\Delta_{i k}^{(0)}=\left|x_{0}(k)-x_{i}(k)\right|$ is absolute difference, $\Delta_{\min }=\min _{s} \min _{t}\left|x_{0}(t)-x_{s}(t)\right|$ is minimum difference of all indices data, $\Delta_{\max }=\max _{s} \max _{t}\left|x_{0}(t)-x_{s}(t)\right|$ is maximum difference of all indices data, and $\rho$ is resolution ration on a scale $[0,1]$.

(5) Calculate the grey correlation degree.

$$
r_{i}=\sum_{k=1}^{n} w_{i} \xi_{i}(k)
$$

Where $w_{i}$ is the weight of every coefficient, and $r_{i}$ is the grey correlation degree.

(6) Analyze the results.

Sort the objective schools in line with the grey correlation degree, and higher ranking represents better performance.

Step 6: Calculate and sort the grey relational coefficient.

To adjust to the amount of investment funding, we set $N$ candidate schools as the upper limit, that is, only the top $N$ schools ranking of the utilization rate of fund have opportunity to attain our investment.

According to above modeling procedure, we get the grey relational coefficient $r_{i}$ of school $i$ by Matlab Programming, and then we sort schools with coefficient $r_{i}$, noting that higher $r_{i}$ relates to higher ranking.

For lack of space, we lists detailed information of the top 10 as selected candidate schools in Table 1.

Table 1 Schools with higher funding utilization rate

\begin{tabular}{cccc}
\hline UNID & r & Duration & Funding sources \\
\hline 155593 & 0.7286 & 2 year & Public \\
212975 & 0.7067 & 2 year & Private \\
413802 & 0.6629 & 2 year & Public \\
191126 & 0.6541 & 2 year & Public \\
202222 & 0.6531 & 2 year & Public \\
188854 & 0.6520 & 2 year & Private \\
206491 & 0.6429 & 4 year & Private \\
161208 & 0.6406 & 2 year & Private \\
179265 & 0.6379 & 4 year & Private \\
219143 & 0.6360 & 2 year & Public \\
\hline
\end{tabular}

\section{Conclusions}

Using the Grey Correlation Analysis method to assess the utilization rate of fund, we avoid large-scale computing the utilization rate directly which strengthens the feasibility of our model. We revise the funding with Consumer Price Index to ensure that same funding can generate same effect 
even in different years, pressing model close to reality. For convenience of selecting appropriate candidate schools for investment, the utilization rate of each school orders by ranking of the grey relational coefficients.

\section{References}

[1]Using Federal Data to Measure and Improve the Performance of US Institutions of Higher Educationhttps://collegescorecard.ed.gov/assets/UsingFederalDataToMeasureAndImprovePerforma nce.pdf

[2]Data Source http://nces.ed.gov/ipeds/datacenter/DataFiles.aspx

[3]Shoukui Si, Xijing Sun. Mathematical Modeling. National Defense Industry Press, 2011,p358-363. 